\title{
Tractable Rational Map Signature
}

\author{
Lih-Chung Wang ${ }^{1, \star}$, Yuh-Hua $\mathrm{Hu}^{2}$, Feipei Lai ${ }^{3}$, \\ Chun-Yen $\mathrm{Chou}^{4, \star \star}$, and Bo-Yin Yang ${ }^{5, \star \star \star}$ \\ 1 Department of Applied Mathematics, \\ National Donghwa University, Hualien 974, Taiwan \\ lcwang@mail.ndhu.edu.tw \\ 2 Department of Computer Science and Information Engineering, \\ National Taiwan University, Taipei 106, Taiwan \\ d92015@csie.ntu.edu.tw \\ 3 Departments of Electrical Engineering \& \\ of Computer Science and Information Engineering, \\ National Taiwan University, Taipei 106, Taiwan \\ flai@ntu.edu.tw \\ 4 Department of Mathematical Education, \\ National Hualien Teachers College, Hualien 970, Taiwan \\ choucy@mail.nhltc.edu.tw \\ 5 Dept. of Mathematics, Tamkang University, Tamsui 251, Taiwan \\ by@moscito.org
}

\begin{abstract}
Digital signature schemes are crucial for applications in electronic commerce. The effectiveness and security of a digital signature scheme rely on its underlying public key cryptosystem. Trapdoor functions are central to public key cryptosystems. However, the modular exponentiation for RSA or the discrete logarithms for ElGamal/DSA/ECC, as the choice of the trapdoor functions, are relatively slow in performance. Some multivariate schemes has potentially much higher performance than other public key cryptosystems. We present a new multivariate digital signature scheme (TRMS) based on tractable rational maps. We also give some security analysis and some actual implementation data in comparison to some other signature schemes.
\end{abstract}

Keywords: multivariate, public key, digital signature, finite field, tractable rational maps

\section{Introduction}

Digital signature schemes are crucial for applications in electronic commerce. For example, to improve the efficiency and maintain the order of stock exchange, each on-line transaction needs to be verified to be validated. The effectiveness and security of a digital signature scheme rely on its underlying public key cryptosystem. Trapdoor functions are central to public key cryptosystems. Only a

\footnotetext{
* Partially supported by National Science Council Grant NSC-93-2115-M-259-003.

** Partially supported by National Science Council Grant NSC-93-2115-M-026-001.

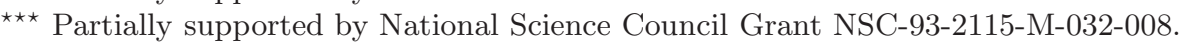


handful of the many schemes attempted reached practical deployment. However, the modular exponentiation for RSA or the discrete logarithms for ElGamal/DSA/ECC, as the choice of the trapdoor functions, are relatively slow in performance. One main reason is the size of the single operand which (at the required security levels) tends to be huge, and this slows the performance.

Some multivariate schemes distinguish themselves from other public key cryptosystems by showing potential for higher performance. For example, Courtois, Goubin and Patarin proposed SFLASH, which has been selected by Nessie Consortium and recommended for low-cost smart cards. The newest version of this signature scheme, SFLASH ${ }^{v 3}$ may be found in [12]. Also, Chen and Yang gave a class of signature (TTS) scheme based on tame transformations in $[4,5,38]$. The newest version of TTS, called Enhanced TTS, outperforms ([40]) all previously known digital signature schemes of comparable security levels, including $\mathrm{SFLASH}^{v 3}$. A summary of this newest instance may be found in [38].

Here we will present a new class of multivariate digital signature scheme (TRMS) based on tractable rational maps. TRMS has similar security and performance as Enhanced-TTS. However there is a small yet non-negligible chance (around 7\%) that signing takes perceptibly longer in the newer versions of TTS. In contrast, the signing time for TRMS is constant, which can do no harm and may be an improvement.

Fix a finite field $\mathbb{K}$ and a natural number $n$. Tractable rational maps on $\mathbb{K}^{n}$ are invertible affine transformations or, after a rearrangement of indices if necessary, functions of the following form $\varphi: \mathbb{K}^{n} \rightarrow \mathbb{K}^{n}$,

$$
\left\{\begin{aligned}
y_{1} & =r_{1}\left(x_{1}\right) \\
y_{2} & =r_{2}\left(x_{2}\right) \frac{p_{2}\left(x_{1}\right)}{q_{2}\left(x_{1}\right)}+\frac{f_{2}\left(x_{1}\right)}{g_{2}\left(x_{1}\right)} \\
& \vdots \\
y_{k} & =r_{k}\left(x_{k}\right) \frac{p_{k}\left(x_{1}, x_{2}, \ldots, x_{k-1}\right)}{q_{k}\left(x_{1}, x_{2}, \ldots, x_{k-1}\right)}+\frac{f_{k}\left(x_{1}, x_{2}, \ldots, x_{k-1}\right)}{g_{k}\left(x_{1}, x_{2}, \ldots, x_{k-1}\right)} \\
& \vdots \\
y_{n}= & r_{n}\left(x_{n}\right) \frac{p_{n}\left(x_{1}, x_{2}, \ldots, x_{n-1}\right)}{q_{n}\left(x_{1}, x_{2}, \ldots, x_{n-1}\right)}+\frac{f_{n}\left(x_{1}, x_{2}, \ldots, x_{n-1}\right)}{g_{n}\left(x_{1}, x_{2}, \ldots, x_{n-1}\right)}
\end{aligned}\right.
$$

where for $i=2,3, \ldots, n, p_{i}, q_{i}, f_{i}, g_{i}$ are polynomials, and for $i=1,2, \ldots, n, r_{i}$ is a permutation polynomial on $\mathbb{K}$. That is, $r_{i}$ is a polynomial function which is also a bijection from $\mathbb{K}$ onto itself.

Let $S=\left\{\left(x_{1}, x_{2}, \ldots, x_{n}\right) \mid \prod_{j=2}^{n} p_{j} q_{j} g_{j} \neq 0\right\}$. For any point in the image set of $S$, it is very easy to find point-wise inverse for tractable rational maps: Given a point $\left(y_{1}, y_{2}, \ldots, y_{n}\right) \in \varphi(S)$, we can easily compute $\left(x_{1}, x_{2}, \ldots, x_{n}\right)$ $\in \mathbb{K}^{n}$ such that $\varphi\left(x_{1}, x_{2}, \ldots, x_{n}\right)=\left(y_{1}, y_{2}, \ldots, y_{n}\right)$. When $\varphi$ is an invertible affine transformation, we can easily write the inverse transformation $\varphi^{-1}$ in an explicit and simultaneous way. That is, we have an explicit formula from which we can compute $x_{1}, x_{2}, \ldots, x_{n}$ simultaneously. When $\varphi$ is not an invertible affine transformation, although it is computationally infeasible to write the inverse in an explicit and simultaneous way, given any point $\left(y_{1}, y_{2}, \ldots, y_{n}\right) \in \varphi(S)$, it 
is very easy to compute $x_{1}, x_{2}, \ldots, x_{n}$ in a sequential way. We simply apply a sequence of substitutions as follows. We refer to this as substitution property.

$$
\left\{\begin{aligned}
x_{1} & =r_{1}^{-1}\left(y_{1}\right) \\
x_{2} & =r_{2}^{-1}\left(\left(y_{2}-\frac{f_{2}\left(x_{1}\right)}{g_{2}\left(x_{1}\right)}\right) \frac{q_{2}\left(x_{1}\right)}{p_{2}\left(x_{1}\right)}\right) \\
& \vdots \\
x_{k} & =r_{k}^{-1}\left(\left(y_{k}-\frac{f_{k}\left(x_{1}, x_{2}, \ldots, x_{k-1}\right)}{g_{k}\left(x_{1}, x_{2}, \ldots, x_{k-1}\right)}\right) \frac{q_{k}\left(x_{1}, x_{2}, \ldots, x_{k-1}\right)}{p_{k}\left(x_{1}, x_{2}, \ldots, x_{k-1}\right)}\right) \\
& \vdots \\
x_{n}= & r_{n}^{-1}\left(\left(y_{n}-\frac{f_{n}\left(x_{1}, x_{2}, \ldots, x_{n-1}\right)}{g_{n}\left(x_{1}, x_{2}, \ldots, x_{n-1}\right)}\right) \frac{q_{n}\left(x_{1}, x_{2}, \ldots, x_{n-1}\right)}{p_{n}\left(x_{1}, x_{2}, \ldots, x_{n-1}\right)}\right)
\end{aligned}\right.
$$

Note that, by Lagrange interpolation, any map over a finite field is a polynomial map. There are both computational and categorical reasons that we put our maps in rational form. For computational reasons, it is faster to compute the division between two function values by low degree polynomial maps than to compute a single function value by a much higher degree polynomial map. For example, it is much easier to compute $\frac{1}{x}$ than to compute $x^{254}$ over $G F(256)$. And categorically, even given a tractable rational map without denominator, by the direct computation above, the inverse of that map is most naturally described as a rational map. Therefore we choose to put the map in the rational form. For details, see [36].

TRMS is the result of exploring the combination of substitution property of tractable rational maps and other mathematical ideas into application of digital signatures.

In [26], T. Moh invented a public key cryptosystem (TTM) based on tame automorphisms which also have the substitution property. It is easily seen that tame transformations are special cases of tractable rational maps with the term $r_{k}\left(x_{k}\right) \frac{p_{k}\left(x_{1}, x_{2}, \ldots, x_{k-1}\right)}{q_{k}\left(x_{1}, x_{2}, \ldots, x_{k-1}\right)}$ replaced by $x_{k}$. Therefore it is not surprising at all that TRMS based on tractable rational maps can achieve similar security and performance as TTS based on tame automorphisms. However, there are also substantial differences between TRMS and TTS with respect to other mathematical ideas and designs.

In section 2, we give the details of TRMS. In section 3, we give some actual implementation data. In section 4, we give some analysis and compare TRMS to other signature schemes, in particular, including TTS.

\section{Details of TRMS}

We show an implement scheme of TRMS. It can be seen that there are a variety of schemes of TRMS which are all based on tractable rational maps.

Let $\mathbb{K}=G F\left(2^{8}\right)$. We will construct 3 maps $\varphi_{1}: \mathbb{K}^{28} \rightarrow \mathbb{K}^{28}, \varphi_{2}: \mathbb{K}^{28} \rightarrow \mathbb{K}^{20}$, $\varphi_{3}: \mathbb{K}^{20} \rightarrow \mathbb{K}^{20}$ where $\varphi_{1}, \varphi_{3}$ are invertible affine transformations, $\varphi_{2}=\pi \circ \widetilde{\varphi_{2}} \circ i$ 
with $\pi$ a projection, $i$ an imbedding, and $\widetilde{\varphi_{2}}$ identified as a tractable rational map over some extension field over $\mathbb{K}$. All the details are given below.

The public key or the verification map $V$ is the result of the composition map $\varphi_{3} \circ \varphi_{2} \circ \varphi_{1}$. Therefore the public key will only be seen as 20 quadratic polynomials in 28 variables whose size is about $8.7 \mathrm{~KB}$ as shown below.

The private key or the key part in the signing map $S$ is the triple $\left(\varphi_{1}, \varphi_{2}, \varphi_{3}\right)$ in some specified structured form whose size is about $0.4 \mathrm{~KB}$ as shown below. As mentioned in the introduction, each $\varphi_{i}$ gives direct instruction to find the point-wise inverse for any concrete instance. Therefore the private key holder or the signer can directly apply $\varphi_{i}^{-1}$ point-wisely.

To sign a message $M$, first find its hash $\mathbf{z}=H(M) \in \mathbb{K}^{20}$ by a publicly agreed hash function. Then do $\mathbf{y}=\varphi_{3}^{-1}(\mathbf{z})$, where the indices of $\mathbf{y}$ is starting at 9 . Then choose 8 nonzero random numbers $r_{1}, r_{2}, \ldots, r_{8}$. Then get $\mathbf{x}$ by identifying it with $\left(\widetilde{\varphi}_{2}^{-1} \circ i\right)\left(r_{1}, r_{2}, \ldots, r_{8}, \mathbf{y}\right)$ which is computed by a sequence of substitutions. Then get the signature $\mathbf{w}=\varphi_{1}^{-1}(\mathbf{x})$.

To verify a signature $\mathbf{w}$, simply check if $V(\mathbf{w})=\left(\varphi_{3} \circ \varphi_{2} \circ \varphi_{1}\right)(\mathbf{w})=\left(\varphi_{3} \circ\right.$ $\left.\pi \circ \widetilde{\varphi_{2}} \circ i\right)(\mathbf{x})=\left(\varphi_{3} \circ \pi\right)\left(r_{1}, r_{2}, \ldots, r_{8}, \mathbf{y}\right)=\varphi_{3}(\mathbf{y})=\mathbf{z}=H(M)$.

\subsection{Details of $\varphi_{1}$ and $\varphi_{3}$}

Since $G F\left(2^{32}\right)$ is finite extension fields of $\mathbb{K}$ of degree 4 , therefore we can identify an element in $\mathbb{K}^{4}$ as an element in $G F\left(2^{32}\right)$. Furthermore, we can decompose $\left(x_{1}, x_{2}, \ldots, x_{28}\right) \in \mathbb{K}^{28}$ into seven groups: for $i=1,2, \ldots, 7, X_{i}=$ $\left(x_{4 i-3}, x_{4 i-2}, x_{4 i-1}, x_{4 i}\right)$ and identify $X_{i} \in G F\left(2^{32}\right), i=1,2, \ldots, 7$. Hence we can identify $\mathbb{K}^{28}$ with $G F\left(2^{32}\right)^{7}$. Similarly, we can identify $\mathbb{K}^{20}$ with $G F\left(2^{32}\right)^{5}$.

Let $\varphi_{1}, \varphi_{3}$ be invertible affine maps on $\mathbb{K}^{28}$ and $\mathbb{K}^{20}$ respectively such that $\varphi_{1}=S_{1} \circ T_{1} \circ L_{1} \circ D_{1} \circ U_{1}$ and $\varphi_{3}=T_{3} \circ L_{3} \circ D_{3} \circ U_{3} \circ S_{3}$ where

1. $S_{1}$ is a circular shift on $\mathbb{K}^{28}$ and $S_{3}$ is a circular shift on $\mathbb{K}^{20}$.

2. $T_{1}$ is a translation on $\mathbb{K}^{28}$ and $T_{3}$ is a translation on $\mathbb{K}^{20} . T_{3}$ is used to cancel the constant terms in the public key. Therefore $T_{3}$ is not chosen but determined.

3. $L_{1}$ is a $7 \times 7$ lower triangular matrix over $G F\left(2^{32}\right)$ and $L_{3}$ is a $5 \times 5$ lower triangular matrix over $G F\left(2^{32}\right)$ such that both with diagonal entries equal to $1 \in G F\left(2^{32}\right)$.

4. $D_{1}$ is a $28 \times 28$ invertible upper triangular matrix over $\mathbb{K}$ and $D_{3}$ is a $20 \times 20$ invertible upper triangular matrix over $\mathbb{K}$ in the following form:

$$
D_{1}=\left(\begin{array}{ccccc}
d_{1} & d_{1}^{2} & d_{1}^{3} & \ldots & d_{1}^{28} \\
0 & d_{2} & d_{2}^{2} & \ldots & d_{2}^{27} \\
0 & 0 & d_{3} & \ldots & d_{3}^{26} \\
\vdots & \vdots & \ddots & \vdots \\
0 & 0 & 0 & \ldots & d_{28}
\end{array}\right)
$$

5. $U_{1}$ is a $7 \times 7$ upper triangular matrix over $G F\left(2^{32}\right)$ and $U_{3}$ is a $5 \times 5$ upper triangular matrix over $G F\left(2^{32}\right)$ such that both with diagonal entries equal to $1 \in G F\left(2^{32}\right)$. 
Note that circular shifts on $\mathbb{K}^{n}$ are indeed linear transformations on $\mathbb{K}^{n}$ and each $T_{i}$ above represents the translation part in the corresponding affine transformation. The $L D U$ decomposition above covers quite a part of general invertible linear transformations. Moreover, our construction enjoys some benefits in key size. With $L_{1}, U_{1}$ linear on $G F\left(2^{32}\right)^{7}$ and $L_{3}, U_{3}$ linear on $G F\left(2^{32}\right)^{5}$, key size of the private key is reduced. Also, the calculation speed of additions is optimized on current 32-bit computer hardware structure. The diagonal entries in $L$ 's and $U$ 's are 1 implies that when we solve $L \mathbf{u}=\mathbf{v}$ or $U \mathbf{u}=\mathbf{v}$ we only have to do additions and multiplications and don't have to bother to do any division. Furthermore, with $D_{1}$ and $D_{3}$ both linear over $\mathbb{K}$ but not on $G F\left(2^{32}\right)$, and also the circular shifts over $\mathbb{K}^{n}$, we can choose $\varphi_{1}, \varphi_{3}$ linear over $\mathbb{K}$, but not linear over $G F\left(2^{32}\right)$. The purpose is to maintain security at the level over $\mathbb{K}$.

\subsection{Details of $\varphi_{2}$}

Let $\mathbb{L}, \mathbb{L}^{\prime}, \mathbb{L}^{\prime \prime}$ be the finite extension fields of $\mathbb{K}$ such that $\mathbb{K} \subset \mathbb{L}^{\prime \prime} \subset \mathbb{L}^{\prime} \subset \mathbb{L}$ and $\left[\mathbb{L}^{\prime \prime}: \mathbb{K}\right]=2, \quad\left[\mathbb{L}^{\prime}: \mathbb{L}^{\prime \prime}\right]=3, \quad\left[\mathbb{L}: \mathbb{L}^{\prime}\right]=3$. Therefore we can identify an element in $\mathbb{K}^{2}$ as an element in $\mathbb{L}^{\prime}=G F\left(2^{16}\right) \subset \mathbb{L}^{\prime} \subset \mathbb{L}$, an element in $\mathbb{K}^{6}$ as an element in $\mathbb{L}^{\prime}=G F\left(2^{48}\right) \subset \mathbb{L}$, and an element in $\mathbb{K}^{18}$ as an element in $\mathbb{L}=G F\left(2^{144}\right)$.

Decompose $\left(x_{1}, x_{2}, \ldots, x_{28}\right) \in \mathbb{K}^{28}$ into five groups: $X_{1}=\left(x_{1}, x_{2}, \ldots, x_{8}\right)$, $X_{2}=\left(x_{9}, x_{10}, x_{11}, x_{12}, x_{13}, x_{14}\right), X_{3}=\left(x_{15}, x_{16}\right), X_{4}=\left(x_{17}, x_{18}, x_{19}\right)$ and $X_{5}=$ $\left(x_{20}, x_{21}, \ldots, x_{28}\right)$. Identify $X_{1}$ with $\left(0, \ldots, 0, x_{1}, x_{2}, \ldots, x_{8}\right) \in \mathbb{L}$. Identify $X_{2} \in$ $\mathbb{K}^{6}$ as an element in $\mathbb{L}^{\prime} \subset \mathbb{L}$. Identify $X_{3} \in \mathbb{K}^{2}$ as an element in $\mathbb{L}^{\prime \prime} \subset \mathbb{L}^{\prime} \subset \mathbb{L}$ and $X_{4} \in \mathbb{K}^{3}$ with $\left(0, x_{17}, 0, x_{18}, 0, x_{19}\right) \in \mathbb{L}^{\prime \prime} \subset \mathbb{L}$. Identify $X_{5} \in \mathbb{K}^{9}$ with $\left(0, x_{20}, 0, x_{21}, \ldots, 0, x_{28}\right)$ as an element in $\mathbb{L}$. Hence we have a natural imbedding $i: \mathbb{K}^{28} \hookrightarrow \mathbb{L}^{5}$ by $i\left(x_{1}, x_{2}, \ldots, x_{28}\right)=\left(X_{1}, X_{2}, X_{3}, X_{4}, X_{5}\right)$. Similarly, decompose $\left(y_{9}, y_{10}, \ldots, y_{32}\right) \in \mathbb{K}^{20}$ into four groups: $Y_{2}=\left(y_{9}, y_{10}, y_{11}, y_{12}, y_{13}, y_{14}\right), Y_{3}=$ $\left(y_{15}, y_{16}\right), Y_{4}=\left(y_{17}, y_{18}, y_{19}\right)$ and $Y_{5}=\left(y_{20}, y_{21}, \ldots, y_{28}\right)$ and identify them as elements in $\mathbb{L}$. For any $r_{i} \in \mathbb{K}, i=1,2, \ldots, 8$, identify $R_{1}=\left(r_{1}, r_{2}, \ldots, r_{8}\right) \in \mathbb{K}^{8}$ with $\left(0, \ldots, 0, r_{1}, r_{2}, \ldots, r_{8}\right) \in \mathbb{L}$. Then we also have

$$
i\left(r_{1}, r_{2}, \ldots, r_{8}, y_{9}, y_{10}, \ldots, y_{28}\right)=\left(R_{1}, Y_{2}, Y_{3}, Y_{4}, Y_{5}\right) \in \mathbb{L}^{5} .
$$

Furthermore, since $\mathbb{K}^{20}$ is a subspace of $\mathbb{L}^{5}=\mathbb{K}^{90}$, we have the projection $\pi$ : $\mathbb{L}^{5} \rightarrow \mathbb{K}^{20}$ such that $(\pi \circ i)\left(r_{1}, r_{2}, \ldots, r_{8}, y_{9}, y_{10}, \ldots, y_{28}\right)=\left(y_{9}, y_{10}, \ldots, y_{28}\right)$

Let $\widetilde{\varphi_{2}}: \mathbb{L}^{5} \rightarrow \mathbb{L}^{5}$ be a tractable rational map of the following form.

$$
\left\{\begin{array}{l}
R_{1}=X_{1} \\
Y_{2}=X_{2} p_{2}\left(X_{1}\right)+f_{2}\left(X_{1}\right) \\
Y_{3}=r_{3}\left(X_{3}\right)+f_{3}\left(X_{1}, X_{2}\right) \\
Y_{4}=X_{4} p_{4}\left(X_{1}, X_{2}, X_{3}\right)+f_{4}\left(X_{1}, X_{2}, X_{3}\right) \\
Y_{5}=X_{5} p_{5}\left(X_{1}, X_{2}, X_{3}, X_{4}\right)+f_{5}\left(X_{1}, X_{2}, X_{3}, X_{4}\right)
\end{array}\right.
$$

such that $\varphi_{2}=\pi \circ \widetilde{\varphi_{2}} \circ i$, and we have the following in $\varphi_{2}$ :

1. $R_{1}=X_{1}$ induces $\left(r_{1}, r_{2}, \ldots, r_{8}\right)=\left(x_{1}, x_{2}, \ldots, x_{8}\right)$.

2. $Y_{2}=X_{2} p_{2}\left(X_{1}\right)+f_{2}\left(X_{1}\right)$ induces 


$$
\left(\begin{array}{c}
y_{9} \\
y_{10} \\
\vdots \\
y_{14}
\end{array}\right)=\left(\begin{array}{c}
x_{9} \\
x_{10} \\
\vdots \\
x_{14}
\end{array}\right) *_{6}\left(\begin{array}{c}
x_{1} \\
x_{2} \\
\vdots \\
x_{6}
\end{array}\right)+\left(\begin{array}{c}
c_{1} x_{1} x_{2} \\
c_{2} x_{2} x_{3} \\
\vdots \\
c_{6} x_{6} x_{7}
\end{array}\right)+\left(\begin{array}{c}
c_{7} x_{3} \\
c_{8} x_{4} \\
\vdots \\
c_{12} x_{8}
\end{array}\right)
$$

where $c_{i}$ 's are constant parameters of user's choice and $\mathbf{u} *_{n} \mathbf{v}$ denotes first identifying $\mathbf{u}, \mathbf{v} \in \mathbb{K}^{n}$ in the extension field with degree $n$ then carrying out the multiplication there. For details see Appendix.

3. $Y_{3}=r_{3}\left(X_{3}\right)+f_{3}\left(X_{1}, X_{2}\right)$ induces

$\left(\begin{array}{l}y_{15} \\ y_{16}\end{array}\right)=\left(\begin{array}{l}x_{15} \\ x_{16}\end{array}\right)^{2}+\left(\begin{array}{c}c_{13} x_{1} x_{2}+c_{14} x_{3} x_{4}+\cdots+c_{19} x_{13} x_{14} \\ c_{20} x_{14} x_{1}+c_{21} x_{2} x_{3}+\cdots+c_{26} x_{12} x_{13}\end{array}\right)+\left(\begin{array}{c}c_{27} x_{1} \\ c_{28} x_{2}\end{array}\right)$

where $\left(\begin{array}{l}x_{15} \\ x_{16}\end{array}\right)^{2}=\left(\begin{array}{l}x_{15} \\ x_{16}\end{array}\right) *_{2}\left(\begin{array}{l}x_{15} \\ x_{16}\end{array}\right)$ and $c_{i}$ 's are constant parameters of user's choice.

4. $Y_{4}=X_{4} p_{4}\left(X_{1}, X_{2}, X_{3}\right)+f_{4}\left(X_{1}, X_{2}, X_{3}\right)$ induces

$$
\left(\begin{array}{l}
y_{17} \\
y_{18} \\
y_{19}
\end{array}\right)=\left(\begin{array}{l}
x_{17} \\
x_{18} \\
x_{19}
\end{array}\right) *_{3}\left(\begin{array}{c}
x_{8} \\
x_{9}+x_{11}+x_{12} \\
x_{13}+x_{15}+x_{16}
\end{array}\right)+\left(\begin{array}{c}
c_{29} x_{4} x_{16} \\
c_{30} x_{5} x_{10} \\
c_{31} x_{15} x_{16}
\end{array}\right)+\left(\begin{array}{c}
c_{32} x_{9} \\
c_{33} x_{10} \\
c_{34} x_{11}
\end{array}\right)
$$

where $c_{i}$ 's are constant parameters of user's choice.

5. $Y_{5}=X_{5} p_{5}\left(X_{1}, X_{2}, X_{3}, X_{4}\right)+f_{5}\left(X_{1}, X_{2}, X_{3}, X_{4}\right)$ induces

$$
\left(\begin{array}{c}
y_{20} \\
y_{21} \\
\vdots \\
y_{28}
\end{array}\right)=\left(\begin{array}{c}
x_{20} \\
x_{21} \\
\vdots \\
x_{28}
\end{array}\right) *\left(\begin{array}{c}
x_{1} \\
x_{2}+x_{6}+x_{11} \\
x_{3}+x_{7}+x_{12} \\
x_{4}+x_{8}+x_{13} \\
x_{5}+x_{9}+x_{14} \\
x_{10}+x_{14}+x_{16} \\
x_{11}+x_{15}+x_{17} \\
x_{12}+x_{16}+x_{18} \\
x_{13}+x_{17}+x_{19}
\end{array}\right)+\left(\begin{array}{c}
c_{35} x_{18} x_{19} \\
c_{36} x_{17} x_{13} \\
c_{37} x_{16} x_{14} \\
c_{38} x_{12} x_{13} \\
c_{39} x_{15} x_{14} \\
c_{40} x_{19} x_{12} \\
c_{41} x_{18} x_{10} \\
c_{42} x_{12} x_{6} \\
c_{43} x_{13} x_{5}
\end{array}\right)+\left(\begin{array}{c}
c_{44} x_{1} \\
c_{45} x_{2} \\
\vdots \\
c_{52} x_{9}
\end{array}\right)
$$

where $c_{i}$ 's are constant parameters of user's choice.

The reason why the formulas in the above assignments represents a permutation polynomial $r_{3}$ and polynomials $p_{2}, f_{2}, f_{3}, p_{4}, f_{4}, p_{5}, f_{5}$ is as follows.

1. We identify $X_{3}=\left(x_{15}, x_{16}\right)$ as an element in $\mathbb{L}^{\prime \prime}=G F\left(2^{16}\right)$ which is of characteristic 2 . For any finite field of characteristic $2, X \mapsto X^{2}$ is an automorphism. Hence let $r_{3}(X)=X^{2}$, then $r_{3}$ is an automorphism on $\mathbb{L}^{\prime \prime}$, hence a permutation polynomial. And $\left(\begin{array}{c}x_{15} \\ x_{16}\end{array}\right) \mapsto\left(\begin{array}{l}x_{15} \\ x_{16}\end{array}\right)^{2}$ surely represents $r_{3}$.

2. For polynomials $p_{2}, f_{2}, f_{3}, p_{4}, f_{4}, p_{5}, f_{5}$, simply notice that on a finite field, any map is a polynomial map. See [36] for details. For example, we show the case of $p_{2}$ for illustration. Consider a map $\mathcal{P}$ on $\mathbb{L}$ as follows 


$$
\mathcal{P}\left(X_{1}\right)=\left\{\begin{array}{c}
0 \\
\vdots \\
0 \\
0 \\
0 \\
x_{1} \\
x_{2} \\
x_{3} \\
x_{4} \\
x_{5} \\
x_{6} \\
\overrightarrow{0} \\
\vec{a}
\end{array}\right. \text { otherwise. }
$$

Simply let $p_{2}$ to be the polynomial representation for $\mathcal{P}$.

It is worth to mention the following.

1. For theoretical purpose we showed above that $\varphi_{2}$ is viewed as $\pi \circ \widetilde{\varphi_{2}} \circ i$ where $\widetilde{\varphi_{2}}: \mathbb{L}^{5} \rightarrow \mathbb{L}^{5}$ is a tractable rational map with polynomials $p_{2}, f_{2}, f_{3}, p_{4}, f_{4}$, $p_{5}, f_{5}$ possibly very complicated. Computationally, we actually follow the other way around. That is, $\varphi_{2}$ is a computationally efficient representation for $\widetilde{\varphi_{2}}$ when restricted to the subspace $i\left(\mathbb{K}^{28}\right)$. We get benefits on calculation speed due the following. The second assignment in $\varphi_{2}$ can be carried out in the subfield $G F\left(2^{48}\right)$ instead of in $\mathbb{L}=G F\left(2^{144}\right)$. For details see appendix. Similarly, the third assignment in $\varphi_{2}$ can be carried out in $\mathbb{L}^{\prime \prime}=G F\left(2^{16}\right)$ instead of in $\mathbb{L}=G F\left(2^{144}\right)$. Both these contribute on calculation speed.

2. It is easily seen that our $\varphi_{2}$ representation is quadratic in $x_{i}$ 's. Since $\varphi_{1}, \varphi_{3}$ are affine maps, the public key is 20 general quadratic polynomials in 28 variables without constant terms.

\subsection{Information on Keys}

As shown above, $\varphi_{1}=S_{1} \circ T_{1} \circ L_{1} \circ D_{1} \circ U_{1}, \varphi_{3}=T_{3} \circ L_{3} \circ D_{3} \circ U_{3} \circ S_{3}$, and there are 52 parameters $c_{1}, c_{2}, \ldots, c_{52}$ for the private key user to choose in $\varphi_{2}$. Therefore the size for private key is $[0+28+4(1+2+3+4+5+6)+28+4(6+$ $5+4+3+2+1)]+[20+4(1+2+3+4)+20+4(4+3+2+1)+0]+52=396$ Bytes. However, $T_{3}$ in $\varphi_{3}$ is not chosen but determined. Hence it is to choose 376 nonzero elements in $\mathbb{K}$ to generate the private key.

Also, since the public key is 20 general quadratic polynomials in 28 variables without constant terms, its size is $20 \cdot\left(\frac{28 \cdot 29}{2}+28\right)=8680$ bytes. In general, there are two ways to generate the public keys. One way is the method of undetermined coefficients, the other one is to make the composition by direct computation. Both have many optimized variants. Our major concern is on the structure of TRMS, therefore we did not put much effort in the optimization of the key generation. 


\section{Performance}

Test Platform: CPU: P4 2.4GHz; RAM: 1024MB; OS: Linux + gcc 3.3; ARG: gcc -O3 -march=pentium4 -fomit-frame-pointer

\begin{tabular}{|c|c|c|c|c|c|c|}
\hline Scheme Name & $\begin{array}{c}\text { Signature } \\
\text { size } \\
(\text { byte })\end{array}$ & $\begin{array}{c}\text { Public } \\
\text { Key Size } \\
(\text { byte })\end{array}$ & $\begin{array}{c}\text { Private } \\
\text { Key Size } \\
(\text { byte })\end{array}$ & $\begin{array}{c}\text { Sign } \\
(\mu \mathrm{s})\end{array}$ & $\begin{array}{c}\text { Key } \\
(\mu \mathrm{s})\end{array}$ & $\begin{array}{c}\text { Generation } \\
(\mathrm{ms})\end{array}$ \\
\hline TTS $(20,28)$ & 28 & 8680 & 1399 & 7 & 20 & 2.2 \\
\hline TRMS $(20,28)$ & 28 & 8680 & 396 & 4.8 & 20 & 1.2 \\
\hline
\end{tabular}

Table: NESSIE signature report, TTS and TRMS tested as above Unit: $\left\{\begin{array}{l}\text { Signature/key size:Bytes, } \\ \text { Sign/Verify/Key Generation: cycles/invocation }\end{array}\right.$

\begin{tabular}{|c|c|c|c|c|c|c|}
\hline Scheme Name & $\begin{array}{c}\text { Signature } \\
\text { size }\end{array}$ & $\begin{array}{c}\text { Public } \\
\text { Key Size }\end{array}$ & $\begin{array}{c}\text { Private } \\
\text { Key Size }\end{array}$ & Sign & Verify & $\begin{array}{c}\text { Key } \\
\text { Generation }\end{array}$ \\
\hline ECDSA & 48 & 48 & 24 & $1971 \mathrm{~K}$ & $5415 \mathrm{~K}$ & $1758 \mathrm{~K}$ \\
\hline ESgin & 144 & 145 & 96 & $4434 \mathrm{~K}$ & $936 \mathrm{~K}$ & $269 \mathrm{M}$ \\
\hline RSA-PSS $^{2}$ & 128 & 128 & 320 & $82 \mathrm{M}$ & $1587 \mathrm{~K}$ & $3206 \mathrm{M}$ \\
\hline SFLASH $_{v 2}$ & 37 & $\approx 15 \mathrm{~K}$ & $\approx 28 \mathrm{~K}$ & $5106 \mathrm{~K}$ & $765 \mathrm{~K}$ & $2929 \mathrm{M}$ \\
\hline SQARTZ $^{16}$ & 16 & $\approx 71 \mathrm{~K}$ & $\approx 4 \mathrm{~K}$ & $6261 \mathrm{M}$ & $144 \mathrm{~K}$ & $3167 \mathrm{M}$ \\
\hline ACESign & 425 & 620 & 748 & $26 \mathrm{M}$ & $20 \mathrm{M}$ & $9645 \mathrm{M}$ \\
\hline TTS $(20,28)$ & 28 & $\approx 8.7 \mathrm{~K}$ & $\approx 1.4 \mathrm{~K}$ & $16.8 \mathrm{~K}$ & $48 \mathrm{~K}$ & $5.28 \mathrm{M}$ \\
\hline TRMS $(20,28)$ & 28 & $\approx 8.7 \mathrm{~K}$ & 396 & $11.4 \mathrm{~K}$ & $48 \mathrm{~K}$ & $2.67 \mathrm{M}$ \\
\hline
\end{tabular}

\section{Analysis and Comparison}

\subsection{Security Analysis}

For brevity, we fix the following notations for our TRMS example:

$-m=20$ denotes the dimension of the hash space.

$-n=28$ denotes the dimension of the signature space.

$-q=2^{8}$ denotes the size of the base field $G F(256)$.

There are several known attacks for multivariate cryptosystems.

Rank Attack: Goubin and Courtois shows that the MinRank attack for Triangular-Plus-Minus systems. Yang and Chen generalized the idea to Rank attack for multivariate systems in [38]. The complexity of the Rank attack is about $q^{r} \cdot \frac{\left(m^{2}\left(\frac{n}{2}-\frac{m}{6}\right)+m n^{2}\right)}{k}$ multiplications, where $k$ is the number of linear combinations of the components of $\varphi_{2}$ which reach the minimal rank $r$. The minimal rank for our example is at least 12 , and $k$ is 6 . Therefore the complexity is about $2^{107}$ multiplications or $2^{101}$ 3DES units ( 1 unit of 3DES $\approx 2^{6}$ multiplications). 
Dual Rank Attack: Coppersmith et al first ([6]) used the Dual Rank attack against multivariate scheme of Shamir; Yang and Chen to generalize this attack to all tame-like multivariate systems in [38]. The complexity of the Dual Rank attack is about $q^{u}\left(u n^{2}+\frac{n^{3}}{6}\right)$ multiplications where $u$ is the minimal number of appearances in $\varphi_{2}$ for any variable $x_{i}$. When $u=9$ for our sample scheme, the complexity is about $2^{86}$ multiplications or $2^{80} 3 \mathrm{DES}$ units.

Unbalanced Oil and Vinegar Attack: As in [38], Let an "oil-set" be any set of independent variables $x_{i}$, such that any of their cross-products never appears in any equation in $\varphi_{2}$. Suppose the maximum size of an oil set is $k$, then then we may determine in time $k^{4} q^{n-2 k-1}$ the "vinegar" and the "oil" subspaces. After that, several possible techniques may be used to find a solution. If case $k=9$, so the time taken to identify the vinegar and oil subspaces is about $2^{86}$ multiplications, or $2^{80}$ 3DES units.

Patarin Relations Attack for $C^{*}$ Family: In $\varphi_{2}$ of our TRMS example, there is no Patarin relation, which means the attack for $C^{*}$ family is not feasible for our system.

Affine Parts Distillation: Geiselmann et al. in [19,20] pointed out the possibility that if the middle portion of any multivariate system is homogeneous of degree two, then it is possible to find the constant parts of both affine mappings easily. The $\varphi_{2}$ in our TRMS example is not homogeneous.

XL Family and Gröbner Bases: Courtois et al proposed the XL method for solving overdetermined quadratic system (which can be viewed as a refinement of the relinearization method by Kipnis-Shamir, [24]) and its variant FXL in [11]. Faugère $([15,16])$ have been improving algorithms for computing Gröbner Bases, and the current state-of-the art variant is $\mathbf{F}_{\mathbf{5}}$, which was used as the critical equation solver in breaking the HFE challenge 1 ([17]). The consensus of current research $([1-3,13,39,41])$ is that Gröbner/XL-like equation solvers on generic equations are exponential in the number of variables. The best variant will be $\mathrm{FF}_{\mathbf{5}}$ if $O\left(n^{2+\varepsilon}\right)$ timing can be achieved, and FXL otherwise. The time complexity for the two methods on a system with $m=20$ equations will be respectively $2^{74}$ and $2^{76}$ 3DES units, still better than RSA-1024 (see [29]). If $m=24$, then we would get $2^{80}$ and $2^{81}$ respectively.

Remark: The speed estimates on nongeneric equations are still being debated, but the converse to Moh's lemma was proved in [39], which shows that it is likely that all Gröbner/XL-like equation solvers will run into trouble if the dimension of the projective solution set at infinity (denoted $\operatorname{dim} H_{\infty}$ ) is non-zero. It is not very easy to benefit from this, however, because the UOV attack means that the last stage of our sample TRMS scheme or something similar cannot be too large, and the dual rank attack dictates that it cannot be too small! Thus for $m=20$, we cannot benefit $\operatorname{dim} H_{\infty}>0$, because the last stage is forced to be 9 variables. For larger TRMS schemes, say $m=28$ upwards, we can start to do better with optimal selection of parameters. 
Finding Minus and Vinegar Variables: These are very specialized methods designed against what is generally called "Big-Field" multivariate schemes such as $C^{*--}$. They do not work against tame-like multivariates with non-constant central parts.

Patarin's IP Approach: Patarin et al proposed an attack method for fixed middle map schemes in $[31,32]$. Since there are variable parameters in the middle map, the IP attack is not applicable.

Search Methods: Courtois et al proposed some search methods at PKC 2002 in [7]. However, they are mainly designed for small finite fields, and we may follow the computations of [4] to find a complexity of $2^{120}$ 3DES units.

\subsection{Comparison to Enhanced-TTS}

The structure of the latest version of TTS, Enhanced-TTS is as follows. Fix a finite field $\mathbb{K}$. Choose three natural numbers $m, n, k$ such that $m<n$ and $k<n-m$. Let $\varphi_{1}, \varphi_{3}$ are invertible affine maps on $\mathbb{K}^{n}$ and $\mathbb{K}^{m}$ respectively. Let $\varphi_{2}: \mathbb{K}^{n} \rightarrow \mathbb{K}^{m}$ be of the following form. (Below $f_{i}$ 's are all quadratic and $\mathbf{y}=\left(y_{n-m+1}, \ldots, y_{n}\right)$.)

$$
\left\{\begin{array}{cl}
r_{1}=x_{1} \\
=x_{2} \\
\vdots & \vdots \\
r_{2} & x_{n-m} \\
r_{n-m} & \left(\begin{array}{c}
\text { invertible } \\
y_{n-m+1} \\
y_{n-m+2} \\
\vdots \\
y_{n-k-j}
\end{array}\right)=\left(\begin{array}{c}
\text { linear } \\
\text { expressions of } \\
x_{1}, \ldots, x_{n-m}
\end{array}\right)\left(\begin{array}{c}
x_{n-m+1} \\
x_{n-m+2} \\
\vdots \\
x_{n-k-j}
\end{array}\right)+\left(\begin{array}{c}
\text { column } \\
\text { vector of } \\
\text { quadratic } \\
\text { expressions of } \\
x_{1}, \ldots, x_{n-m}
\end{array}\right) \\
= & x_{n-k-j+1}+f_{n-k-j+1}\left(x_{1}, x_{2}, \ldots, x_{n-k-j}\right) \\
& \left.\vdots \begin{array}{c}
\text { invertible } \\
y_{n-k} \\
y_{n-k+1} \\
y_{n-k+2} \\
\vdots \\
y_{n}
\end{array}\right)=\left(\begin{array}{c}
\text { linearix of } \\
\text { expressions of } \\
x_{1}, \ldots, x_{n-k}
\end{array}\right)\left(\begin{array}{c}
x_{n-k+1} \\
x_{n-k+2} \\
\vdots \\
x_{n}
\end{array}\right)+\left(\begin{array}{c}
\text { column } \\
\text { quadratic } \\
\text { expressions of } \\
x_{1}, \ldots, x_{n-k}
\end{array}\right)
\end{array}\right.
$$

The verification map $V$ can be decomposed as $\mathbf{w} \in \mathbb{K}^{n} \stackrel{\varphi_{1}}{\longmapsto} \mathbf{x} \stackrel{\varphi_{2}}{\longmapsto} \mathbf{y} \stackrel{\varphi_{3}}{\longmapsto} \mathbf{z} \in \mathbb{K}^{m}$. That is, $V=\varphi_{3} \circ \varphi_{2} \circ \varphi_{1}$, where $\mathbf{x}=\varphi_{1}(\mathbf{w})=\mathrm{M}_{1} \mathbf{w}+\mathbf{c}_{1}, \mathbf{z}=\varphi_{3}(\mathbf{y})=\mathrm{M}_{3} \mathbf{y}+\mathbf{c}_{3}$ and $\left(r_{1}, r_{2}, \ldots, r_{n-m}, \mathbf{y}\right)=\varphi_{2}(\mathbf{x})$.

To sign a message, Enhanced-TTS needs to solve two systems of equations for finding one inverse image point of the middle map. There is about $1 / 25$ chance of redoing the signing procedure for the implement in [38]. However, our TRMS example has constant signing time, since the non-zero element in a field is always invertible. 
Regarding to signing time, TRMS is better than TTS. One reason is that TRMS utilizes special field extension structure to reduce the computation time for $\varphi_{2}^{-1}$, the details is in the Appendix, while TTS only uses the common method of Gaussian elimination. Another reason is that during computation of the affine transformations $\varphi_{1}, \varphi_{3}$, part of it is also carried out in a larger field, which will benefit the computation, too. We like to point out that there are a lot of ways to construct $\varphi_{1}, \varphi_{3}$. One reason for us to use the $L U$-decomposition is that it has advantages when implemented on smart cards.

The main external differences between TRMS(20,28) and EnhancedTTS $(20,28)$ can be tabulated as follows.

1. The private key size for TTS is $1.4 \mathrm{~KB}$, while for TRMS it is 396 bytes.

2. Regarding to signing time, TRMS is better than TTS.

3. TTS has at most $7 \%$ chance of redoing the signing procedure while the signing time for TRMS is constant.

\section{Appendix: Implement of Field Extension}

Firstly, $G F(2)=\left\{(0)_{2},(1)_{2}\right\}$, where $(\cdot)_{2}$ means the binary representation. Then $t^{2}+t+(1)_{2}$ is irreducible over $G F(2)$. Let $G F(4)=G F(2)[t] /\left(t^{2}+t+(1)_{2}\right)$ and $(a b)_{2}$ denote the equivalent class of $a t+b$. Then we have the following multiplication table.

\begin{tabular}{|l|l|l|l|l|}
\hline & $(00)_{2}$ & $(01)_{2}$ & $(10)_{2}$ & $(11)_{2}$ \\
\hline$(00)_{2}$ & $(00)_{2}$ & $(00)_{2}$ & $(00)_{2}$ & $(00)_{2}$ \\
\hline$(01)_{2}$ & $(00)_{2}$ & $(01)_{2}$ & $(10)_{2}$ & $(11)_{2}$ \\
\hline$(10)_{2}$ & $(00)_{2}$ & $(10)_{2}$ & $(11)_{2}$ & $(01)_{2}$ \\
\hline$(11)_{2}$ & $(00)_{2}$ & $(11)_{2}$ & $(01)_{2}$ & $(10)_{2}$ \\
\hline
\end{tabular}

Similarly, we have $t^{2}+t+(10)_{2}$ is irreducible over $G F(4)$. Let $G F(16)=$ $G F(4)[t] /\left(t^{2}+t+(10)_{2}\right)$ and $(a b c d)_{2}$ denote the equivalent class of $(a b)_{2} t+(c d)_{2}$. Then we can construct a multiplication table of size $16 \times 16$.

Similarly, we have $t^{2}+t+(1000)_{2}$ is irreducible over $G F(16)$. Let $G F(256)=$ $G F(16)[t] /\left(t^{2}+t+(1000)_{2}\right)$ and $(a b c d e f g h)_{2}$ denote the equivalent class of $(a b c d)_{2} t+(e f g h)_{2}$. Then we can construct a multiplication table of size $256 \times 256$.

Similarly, we have $t^{2}+t+(1000,0000)_{2}$ is irreducible over $G F(256)$. Let $\alpha_{1}=(1000,0000)_{2}$. Let $G F\left(2^{16}\right)=G F(256)\left[t_{1}\right] /\left(t_{1}^{2}+t_{1}+\alpha_{1}\right)$. However, we do not construct the multiplication table of $G F\left(2^{16}\right)$. For $a, b, c, d \in G F(256)$, $\left(a t_{1}+b\right)\left(c t_{1}+d\right)=a c t_{1}^{2}+(a d+b c) t_{1}+b d=a c\left(t_{1}+\alpha_{1}\right)+(a d+b c) t_{1}+b d=$ $[(a+b)(c+d)+b d] t_{1}+\left[a c \alpha_{1}+b d\right]$.

Similarly, we have $t^{2}+t+(1000,0000,0000,0000)_{2}$ is irreducible over $G F\left(2^{16}\right)$. Let $\alpha_{2}=(1000,0000,0000,0000)_{2}$. Let $G F\left(2^{32}\right)=G F\left(2^{16}\right)\left[t_{2}\right] /\left(t_{2}^{2}+t_{2}+\alpha_{2}\right)$. For $A, B, C, D \in G F\left(2^{16}\right),\left(A t_{2}+B\right)\left(C t_{2}+D\right)=[(A+B)(C+D)+B D] t_{2}+$ $\left[A C \alpha_{2}+B D\right]$.

Note that we now have a recursive definition for $G F\left(\left(2^{8}\right)^{\left(2^{i}\right)}\right)$. With a proper choice of $\alpha_{i}$, we let $G F\left(\left(2^{8}\right)^{\left(2^{i}\right)}\right)=G F\left(\left(2^{8}\right)^{\left(2^{i-1}\right)}\right)\left[t_{i}\right] /\left(t_{i}^{2}+t_{i}+\alpha_{i}\right)$. For $a, b, c, d \in$ $G F\left(\left(2^{8}\right)^{\left(2^{i-1}\right)}\right)$, 


$$
\left(a t_{i}+b\right)\left(c t_{i}+d\right)=[(a+b)(c+d)+b d] t_{i}+\left[a c \alpha_{i}+b d\right]
$$

where the addition is the bitwise XOR and the multiplication of expressions of $a, b, c, d$ and $\alpha_{i}$ are done in $G F\left(\left(2^{8}\right)^{\left(2^{i-1}\right)}\right)$.

To find the inverse of $a t_{i}+b$, first we let $\left(a t_{i}+b\right)\left(A t_{i}+B\right)=1$, that is, $(a A+a B+A b) t_{i}+a A \alpha_{i}+b B=1$ or, in vector form, by considering $\left\{t_{i}, 1\right\}$ as a basis, $\left(\begin{array}{cc}a+b & a \\ a \alpha_{i} & b\end{array}\right)\left(\begin{array}{l}A \\ B\end{array}\right)=\left(\begin{array}{l}0 \\ 1\end{array}\right)$. Hence $\left(\begin{array}{l}A \\ B\end{array}\right)=\left(\begin{array}{cc}a+b & a \\ a \alpha_{i} & b\end{array}\right)^{-1}\left(\begin{array}{l}0 \\ 1\end{array}\right)=$ $\left(a b+b^{2}+a^{2} \alpha_{i}\right)^{-1}\left(\begin{array}{cc}b & a \\ a \alpha_{i} & a+b\end{array}\right)\left(\begin{array}{l}0 \\ 1\end{array}\right)=\left(a b+b^{2}+a^{2} \alpha_{i}\right)^{-1}\left(\begin{array}{c}a \\ a+b\end{array}\right)$. Therefore $\left(a t_{i}+b\right)^{-1}=\left(a t_{i}+a+b\right)\left(a b+b^{2}+a^{2} \alpha_{i}\right)^{-1}$.

Here we give an example of field extension of degree 12 to illustrate how we can accelerate the computation of large field. We let $\mathbb{K}=G F\left(2^{8}\right)$ and $\mathbb{L}, \mathbb{L}^{\prime}, \mathbb{L}^{\prime \prime}$ be the finite extension fields of $\mathbb{K}$ such that $\mathbb{K} \subset \mathbb{L}^{\prime \prime} \subset \mathbb{L}^{\prime} \subset \mathbb{L}$ and $\left[\mathbb{L}^{\prime \prime}: \mathbb{K}\right]=2$, $\left[\mathbb{L}^{\prime}: \mathbb{L}^{\prime \prime}\right]=2, \quad\left[\mathbb{L}: \mathbb{L}^{\prime}\right]=3$. Therefore $\mathbb{L}^{\prime}=G F\left(2^{16}\right), \mathbb{L}^{\prime}=G F\left(2^{32}\right) \subset \mathbb{L}$, and $\mathbb{L}=G F\left(2^{96}\right)$ and we need to discuss the field extension of degree 3 below.

Since $t^{3}+t+1$ is irreducible over $G F\left(2^{32}\right)[t]$, we can identify $G F\left(2^{96}\right)$ with $G F\left(2^{32}\right)[t] /\left(t^{3}+t+1\right)$. If we use $\left(\begin{array}{l}a \\ b \\ c\end{array}\right)$ to represent $a t^{2}+b t+c$, then

$$
\left(\begin{array}{l}
a \\
b \\
c
\end{array}\right) * 12\left(\begin{array}{l}
x_{1} \\
x_{2} \\
x_{3}
\end{array}\right)=\left(\begin{array}{ccc}
(a+c) & b & a \\
(a+b) & (a+c) & b \\
b & a & c
\end{array}\right)\left(\begin{array}{l}
x_{1} \\
x_{2} \\
x_{3}
\end{array}\right)
$$

where $*_{12}$ denotes the multiplication in $\mathbb{L}$ and the right hand side is just the usual matrix multiplication. In signing a message, we need to solve $\mathbf{a x}=\mathbf{y}$ for $\mathbf{x}$ in $\mathbb{L}$. That is, to solve

$$
\left(\begin{array}{ccc}
(a+c) & b & a \\
(a+b) & (a+c) & b \\
b & a & c
\end{array}\right)\left(\begin{array}{l}
x_{1} \\
x_{2} \\
x_{3}
\end{array}\right)=\left(\begin{array}{l}
y_{1} \\
y_{2} \\
y_{3}
\end{array}\right)
$$

for $x_{1}, x_{2}, x_{3}$. Therefore, we have

$$
\left(\begin{array}{l}
x_{1} \\
x_{2} \\
x_{3}
\end{array}\right)=\frac{1}{\triangle}\left[\operatorname{adj}\left(\begin{array}{ccc}
(a+c) & b & a \\
(a+b) & (a+c) & b \\
b & a & c
\end{array}\right)\right]\left(\begin{array}{l}
y_{1} \\
y_{2} \\
y_{3}
\end{array}\right) .
$$

Write out adj $\left(\begin{array}{ccc}(a+c) & b & a \\ (a+b) & (a+c) & b \\ b & a & c\end{array}\right)$ as $\left(\begin{array}{ccc}A_{11} & A_{12} & A_{13} \\ A_{21} & A_{22} & A_{23} \\ A_{31} & A_{32} & A_{33}\end{array}\right)$, then

$$
\begin{aligned}
& A_{31}=A_{12}=A_{23}=a^{2}+b c \\
& A_{11}=A_{22}=a(b+c)+c^{2} \\
& A_{32}=A_{13}=a c+(a+b)^{2} \\
& A_{21}=A_{31}+A_{13} \\
& A_{33}=A_{22}+A_{32} \\
& \triangle=a A_{31}+b A_{32}+c A_{33}
\end{aligned}
$$

According to the calculation above, to solve $\mathbf{a x}=\mathbf{y}$, we need 21 multiplications and one inverse operation in $G F\left(2^{32}\right)$, which is roughly 342 multiplications 
in $\mathbb{K}$. Comparing to TTS, doing the Gaussian elimination for two $9 \times 9$ matrices, it takes at least about $2 \times 9^{3} / 3 \approx 500$ multiplications in $\mathbb{K}$.

Note: There will be a extended version at IACR eprint archive.

\section{References}

1. G. Ars and J.-C. Faugère, Comparison of XL and Gröbner Bases Algorithms over Finite Fields, preprint. Will appear as one half of an article at Asiacrypt 2004 and LNCS.

2. M. Bardet, J.-C. Faugère, and B. Salvy, Complexity of Gröbner Basis Computations for Regular Overdetermined Systems, INRIA Rapport de Recherche No. 5049; a slightly modified preprint is accepted by the International Conference on Polynomial System Solving.

3. M. Bardet, J.-C. Faugère, B. Salvy, and B.-Y. Yang, Asymptotic Complexity of Gröbner Basis Algorithms for Semi-regular Overdetermined Systems over Large Fields, manuscript in preparation.

4. J.-M. Chen and B.-Y. Yang, Tame Transformations Signatures With Topsy-Turvy Hashes, proc. IWAP 2002, Taipei.

5. J.-M. Chen and B.-Y. Yang, A More Secure and Efficacious TTS Scheme, ICISC 2003, LNCS v. 2971, pp. 320-338; full version at eprint.iacr.org/2003/160.

6. D. Coppersmith, J. Stern, and S. Vaudenay, Attacks on the Birational Permutation Signature Schemes, Crypto 1993, LNCS v. 773, pp. 435-443.

7. N. Courtois, L. Goubin, W. Meier, and J. Tacier, Solving Underdefined Systems of Multivariate Quadratic Equations, PKC 2002, LNCS v. 2274, pp. 211-227

8. N. Courtois, Generic Attacks and the Security of Quartz, PKC 2003, LNCS v. 2567, pp. 351-364.

9. N. Courtois, Algebraic Attacks over $G F\left(2^{k}\right)$, Cryptanalysis of HFE Challenge 2 and SFLASH ${ }^{v 2}$, accepted for PKC 2004.

10. N. Courtois, A. Klimov, J. Patarin, and A. Shamir, Efficient Algorithms for Solving Overdefined Systems of Multivariate Polynomial Equations, EUROCRYPT 2000, LNCS v. 1807, pp. 392-407.

11. N. Courtois and J. Patarin, About the XL Algorithms over GF(2), CT-RSA 2003, LNCS v. 2612, pp. 141-157.

12. N. Courtois, L. Goubin, and J. Patarin, SFLASH ${ }^{3}$, a Fast Asymmetric Signature Scheme, preprint

13. C. Diem, The XL-algorithm and a Conjecture from Commutative Algebra, preprint (to appear Asiacrypt 2004 and LNCS) and private communication.

14. W. Diffie and M. Hellman, New Directions in Cryptography, IEEE Trans. Info. Theory, vol. IT-22, no. 6, pp. 644-654.

15. J.-C. Faugére, A New Efficient Algorithm for Computing Gröbner Bases (F4), Journal of Pure and Applied Algebra, 139 (1999), pp. 61-88.

16. J.-C. Faugère, A New Efficient Algorithm for Computing Gröbner Bases without Reduction to Zero (F5), Proc. ISSAC 2002, pp. 75-83, ACM Press 2002.

17. J.-C. Faugère and A. Joux, Algebraic Cryptanalysis of Hidden Field Equations (HFE) Cryptosystems Using Gröbner Bases, Crypto 2003, LNCS v. 2729, pp. 44-60.

18. M. Garey and D. Johnson, Computers and Intractability, A Guide to the Theory of NP-completeness, 1979, p. 251.

19. W. Geiselmann, R. Steinwandt, and T. Beth, Attacking the Affine Parts of SFLASH, 8th International IMA Conference on Cryptography and Coding, LNCS v. 2260, pp. 355-359. 
20. W. Geiselmann, R. Steinwandt, and T. Beth, Revealing the 441 Key Bits of SFLASH ${ }^{v 2}$, Third NESSIE Workshop, 2002.

21. L. Goubin and N. Courtois, Cryptanalysis of the TTM cryptosystem, Asiacrypt 2000, LNCS v. 1976, pp. 44-57.

22. A. Kipnis and A. Shamir, Cryptanalysis of the Oil and Vinegar Signature Scheme, Crypto'98, LNCS v. 1462, pp. 257-266

23. A. Kipnis, J. Patarin, and L. Goubin, Unbalanced Oil and Vinegar Sigature Schemes, Crypto'99, LNCS v. 1592, pp. 206-222

24. A. Kipnis and A. Shamir, Cryptanalysis of the HFE Public Key Cryptosystem by Relinearization, Crypto'99, LNCS v. 1666, pp. 19-30

25. T. Matsumoto and H. Imai, Public Quadratic Polynomial-Tuples for Efficient Signature-Verification and Message-Encryption, EUROCRYPT'88, LNCS v. 330, pp. 419-453.

26. T. Moh, A Public Key System with Signature and Master Key Functions, Communications in Algebra, 27 (1999), pp. 2207-2222.

27. T. Moh and J. -M. Chen, On the Goubin-Courtois Attack on TTM, published electronically by Cryptology ePrint Archive (2001/072).

28. New European Schemes for Signatures, Integrity, and Encryption, project homepage at http://www.cryptonessie.org.

29. Performance of Optimized Implementations of the NESSIE primitives, version 2.0 http://www.cryptonessie.org.

30. J. Patarin, Cryptanalysis of the Matsumoto and Imai Public Key Scheme of Eurocrypt'88, Crypto'95, LNCS v. 963, pp. 248-261.

31. J. Patarin, Hidden Fields Equations (HFE) and Isomorphisms of Polynomials (IP) Two New Families of Asymmetric Algorithms, EUROCRYPT'96, LNCS v. 1070, pp. 33-48.

32. J. Patarin, L. Goubin, N. Courtois, Improved Algorithm for Isomorphisms of Polynomials, EUROCRYPT'98, LNCS v. 1403, pp. 184-200.

33. J. Patarin, N. Courtois, and L. Goubin, QUARTZ, 128-Bit Long Digital Signatures, CT-RSA 2001, LNCS v. 2020, pp. 282-297. Updated version available at http://www.cryptonessie.org.

34. J. Patarin, N. Courtois, and L. Goubin, FLASH, a Fast Multivariate Signature Algorithm, CT-RSA 2001, LNCS v. 2020, pp. 298-307. Updated version available at http://www.cryptonessie.org.

35. A. Shamir and E. Tromer, Factoring Large Numbers with the TWIRL Device, Crypto 2003, LNCS v. 2729, pp. 1-26.

36. Lih-Chung Wang and Fei-Hwang Chang, Tractable Rational Map Cryptosystem, available at http://eprint.iacr.org/2004/046.

37. C. Wolf, Efficient Public Key Generation for Multivariate Cryptosystems, preprint, available at http://eprint.iacr.org/2003/089.

38. B.-Y. Yang and J.-M. Chen, Rank Attacks and Defence in Tame-Like Multivariate PKC's, see http://eprint.iacr.org/2004/061.

39. B.-Y. Yang and J.-M. Chen, All in the XL Family: Theory and Practice, to appear at ICISC 2004 and LNCS.

40. B.-Y. Yang, Y.-H. Chen, and J.-M. Chen, TTS: High-Speed Signatures on a Low-End Smart Card, Proc. CHES '04, LNCS v. 3156, pp. 371-385.

41. B.-Y. Yang, J.-M. Chen, and N. Courtois, On Asymptotic Security Estimates in XL and Gröbner Bases-Related Algebraic Cryptanalysis, ICICS 2004, LNCS v. 3269, pp. 401-413. 